\title{
Transformation of Broca's aphasia into conduction aphasia: a case report
}

\author{
S. Kazui, T. Sawada, H. Tanabe ${ }^{1}$ and N. Inoue \\ Cerebrovascular Division, Department of Medicine, National Cardiovascular Center, \\ Osaka, and 'Department of Neuropsychiatry, Osaka University, Osaka, Japan \\ Correspondence to: S. Kazui, Cerebrovascular Division, Department of Medicine, \\ National Cardiovascular Center, 5-7-1 Fujishirodai, Suita, Osaka 565, Japan
}

\begin{abstract}
We describe a patient with a cerebral infarction localized mainly in the left posterior frontal and anterior parietal subcortices who experienced a Broca's aphasia which evolved into a conduction aphasia. Such a rare recovery pattern of aphasia appeared to result from amelioration of the damage to the left precentral gyrus.
\end{abstract}

Keywords: Arcuate fasciculus - Broca's aphasia - Conduction aphasia - Precentral gyrus

\section{INTRODUCTION}

Some patients with Wernicke's aphasia during recovery develop a conduction aphasia (Kertesz, 1984). To the best of our knowledge, however, evolution from Broca's aphasia to conduction aphasia has not yet been described. We recently experienced a patient with Broca's aphasia whose language disorder transformed into conduction aphasia. This report describes the pertinent clinical and neuroradi-

ological findings.

\section{CASE REPORT}

A 63-year-old right-handed woman with a history of aortic valve replacement for aortic stenosis 4 years previously, suddenly developed language difficulties and motor weakness of the right arm when she was doing house work on 29 December 1989.

On admission, the patient was alert with a good insight into her illness. Her blood pressure was $164 / 84 \mathrm{mmHg}$. Her pulse was 90 beats/min without arrhythmia. The results of physical examination were normal except for metallic heart sounds due to the valve prosthesis. Neurologic examinations disclosed a right hemiparesis involving the face and with a predominance of arm weakness, and co-existent hypesthesia down the right side. The deep tendon reflexes were exaggerated and the plantar response was extensor on the right. Her verbal output was sparse, poorly articulated and dysprosodic. Her spontaneous speech was produced with considerable effort, particularly on initiation, and consisted of very short phrases which were telegraphic. There were some phonemic (literal) paraphasias in her spontaneous speech. Comprehension of spoken language was considerably better than verbal output; three-step verbal commands were correctly executed. Repetition of spoken words was defective with articulatory and phonemic problems, as was confrontation naming, in which word-finding difficulty was also noted. Writing was abnormal especially in Kana, showing poorly formed letters, literal paragraphias and misspellings. The patient had buccofacial apraxia but no ideomotor apraxia. Mild constructional apraxia was observed. Calculations were assessed as fairly good. There was no evidence of finger agnosia or right-left confusion. Unilateral spatial neglect and extinction phenomena were absent.

The right hemiparesis rapidly ameliorated, and only mild difficulties in discrete movements remained 1 month after onset. In parallel with the regression of the motor and sensory deficits, the articulatory problems in speech cleared up and spontaneous speech became fluent within a month. In conversation, there were mild word-finding difficulties and occasional phonemic paraphasias recognized as errors by the patient. Repetition of verbal material was, however, impaired with frequent phonemic paraphasias, accompanied by groping for phonemes and target approximations (conduites d'approche). Comprehension was near-normal. Although the writing disturbance also improved, sufficient data could not be obtained for detailed evaluation because of the patient's disinclination to write. Buccofacial apraxia and mild constructional apraxia still remained.

Behavioural Neurology . Vol 5 . 1992 


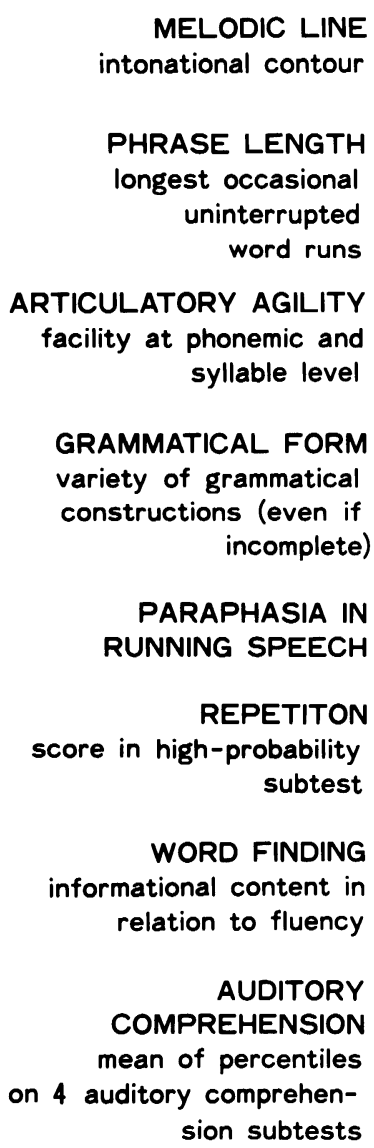

MELODIC LINE

PHRASE LENGTH

est occasional

ninterrupted

word runs

ARTICULATORY AGILITY

phonemic and

syllable level

GRAMMATICAL FORM variety of grammatical ins (even if

PARAPHASIA IN

REPETITON

subtest

WORD FINDING

relation to fluency

AUDITORY

COMPREHENSION

sion subtests

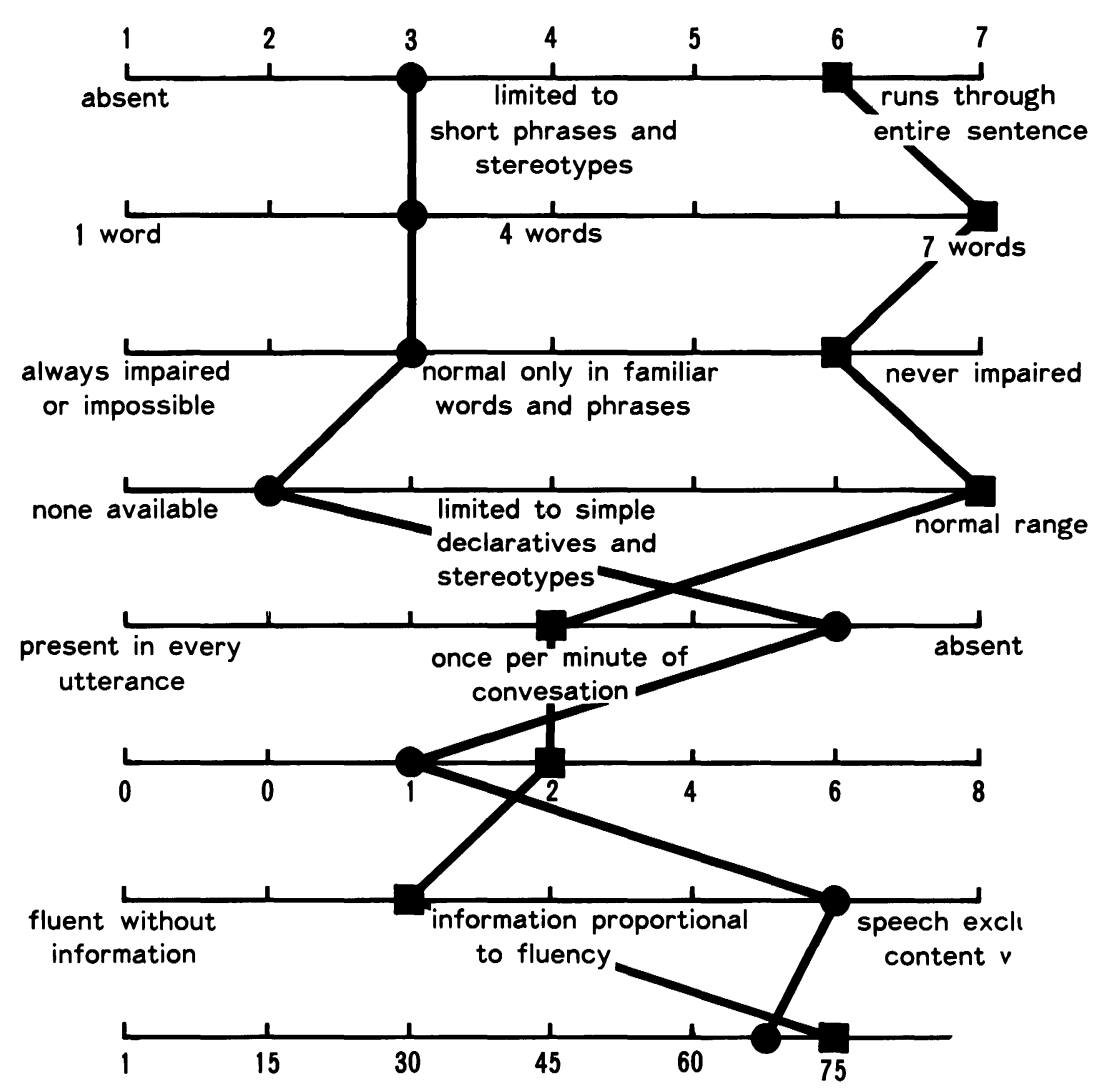

7 days; 1 month after onset.
The rating scale profiles of the patient's speech characteristics are presented in Fig. 1 for 7 days and 1 month after onset. The pattern in the initial stage is consistent with Broca's aphasia, and that in the recovery stage, with conduction aphasia (Goodglass and Kaplan, 1983).

\section{NEURORADIOLOGICAL FINDINGS}

Computed axial tomography (CT scan) at 42 days after onset revealed hypodense areas in the posterior frontal and anterior parietal lobes of the left cerebral hemisphere (Fig. $2)$. The anterior lesion involved the subcortex of the precentral gyrus and small aspects of the anterior insula. The posterior lesion encroached upon the subcortex of the postcentral gyrus, the arcuate fasciculus and the corona radiata. The inferior frontal gyrus was almost intact and the supramarginal gyrus was spared. Magnetic resonance imaging could not be carried out because of the mechanical valve in the patient's heart. A left carotid angiogram on admission revealed occlusion of the upper trunk of the left middle cerebral artery (MCA), which was recanalized on repeat angiography 11 days later (Fig. 3). Single photon emission computed tomography (SPECT) at 12 days after onset showed decreased accumulation of $N$-isopropyl- $p$ ( ${ }^{123} \mathrm{I}$ )-iodo-amphetamine (IMP) in the entire territory of the left MCA (Fig. 4).

\section{DISCUSSION}

The initial speech characteristics in our patient coincided with the clinical picture of Broca's aphasia: non-fluent verbal output, relatively intact comprehension, and abnormalities in repetition of spoken language, confrontation naming and writing (Benson, 1979). Phonetic disintegration (Alajouanine, 1956) was quite characteristic in the patient. Mori and colleagues (1989) reported a case of Broca's aphasia caused by restricted damage to the left lower precentral gyrus. The subcortical lesion of the precentral gyrus seemed to play an important role in the development of the phonetic disintegration in our patient. In addition, the speech characteristics of Broca's aphasia at the acute stage might be related to a decreased cerebral blood flow in the left anterior MCA territory as was verified by the initial cerebral angiography. In parallel with the amelioration of the right-sided hemiparesis and hypesthesia, the disorders of verbal output and articulation 
A

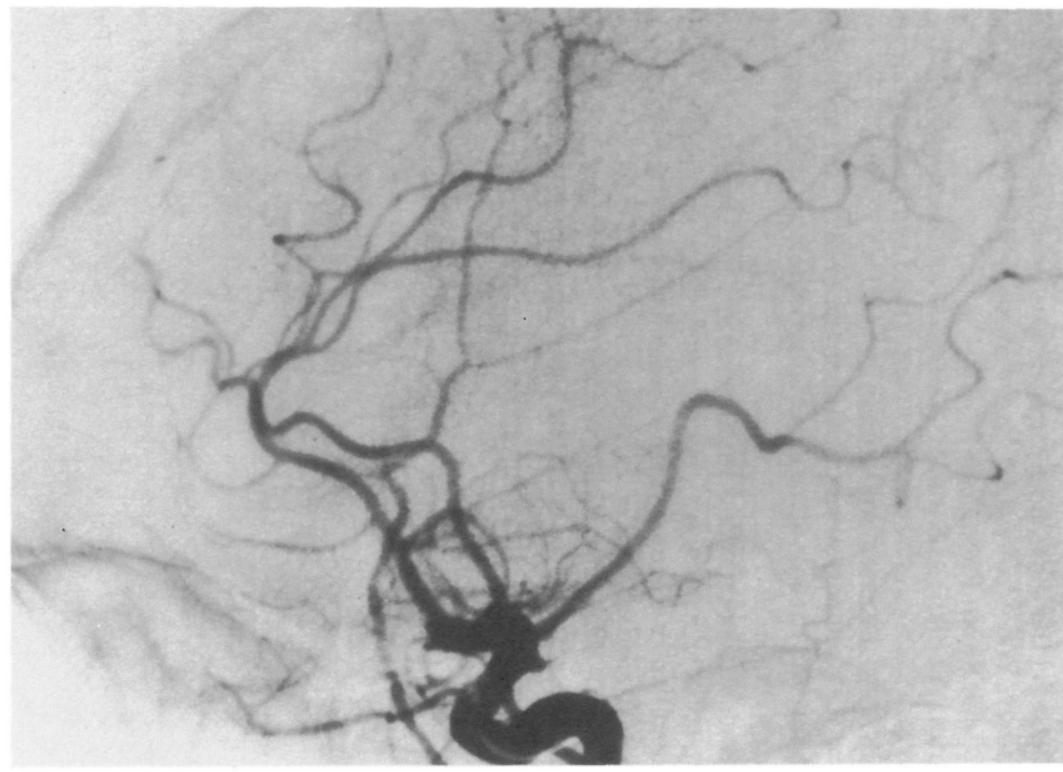

B

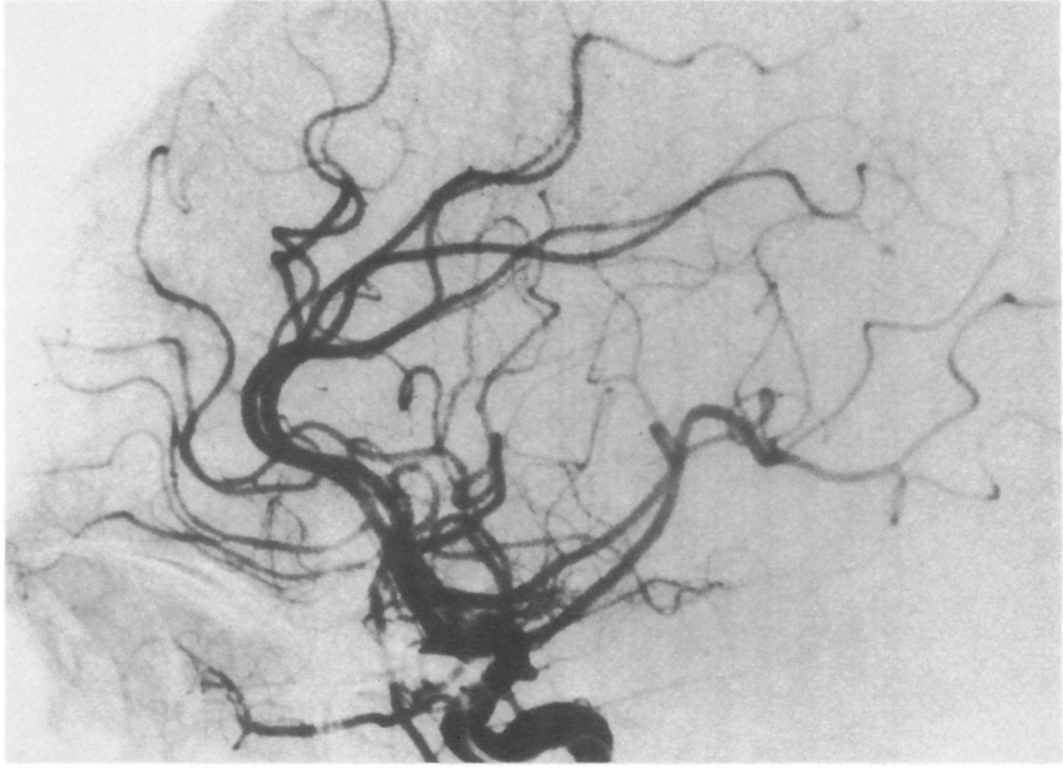

FIG. 3. Left carotid angiograms revealing occlusion of the upper trunk of the left middle cerebral artery on admission (A), which was reopened 11 days later (B).
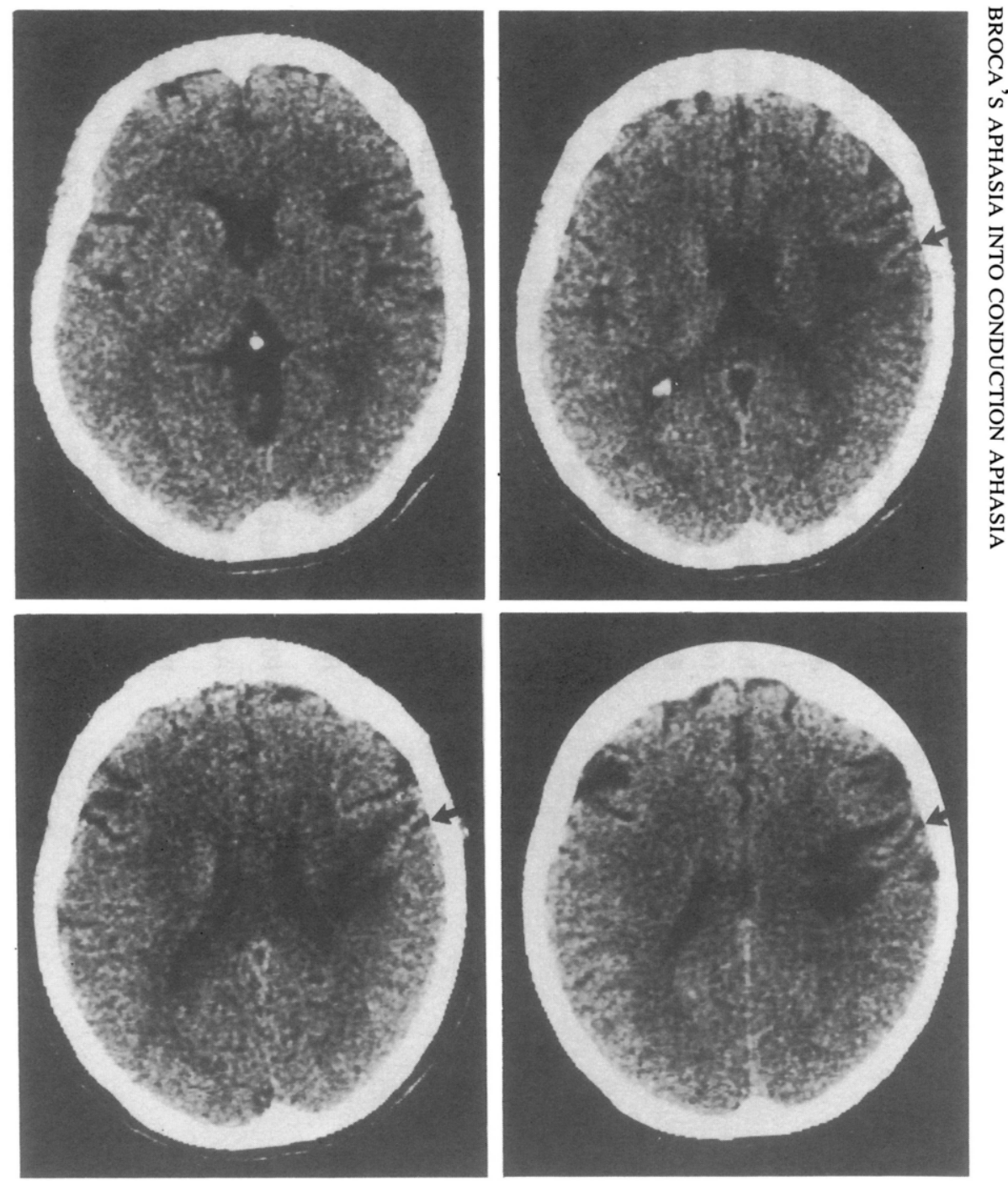

$\leftarrow$ C.S.

FIG. 2. CT scan. Hypodense areas are seen mainly in the subcortices of the left posterior frontal and anterior parietal regions. C.S., central sulcus. 


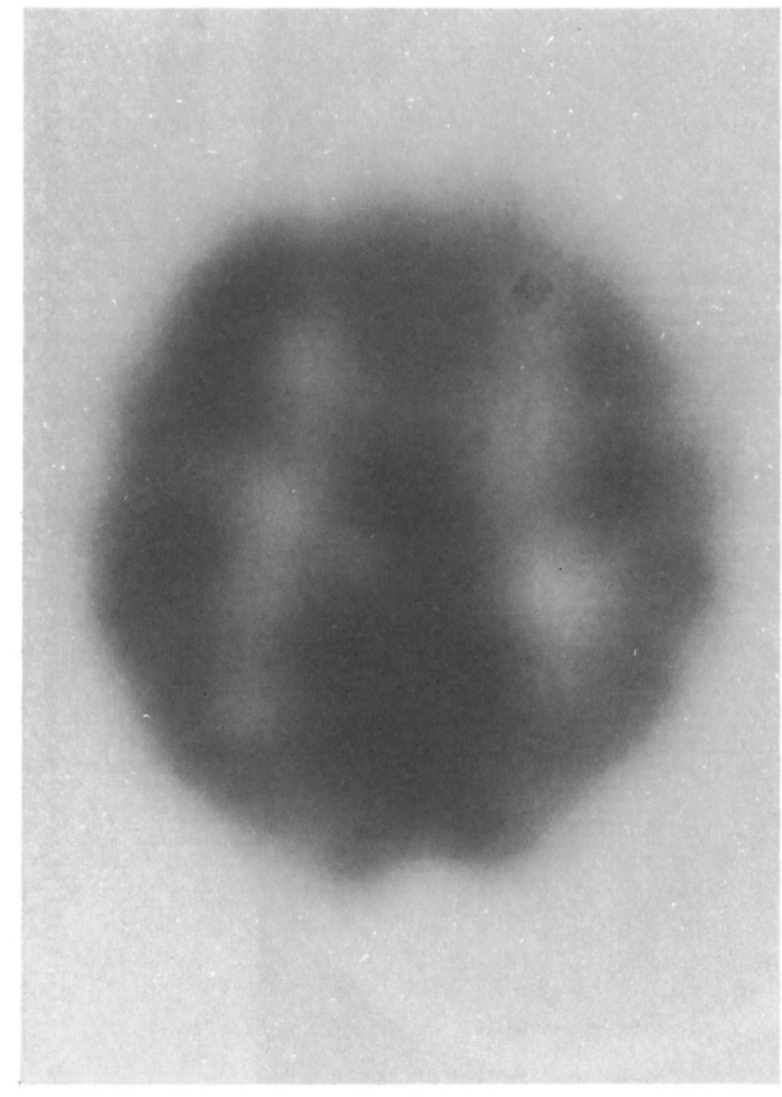

FIG. 4. SPECT at the acute stage showing decreased accumulation of ${ }^{123}$-IMP in the left middle cerebral artery territory.

rapidly improved. Thus, at 1 month after onset, our patient exhibited fluent paraphasic speech with markedly impaired repetition of words and phrases with attempts to correct, which represent the primary features of conduction aphasia (Benson et al., 1973). Kertesz (1985) stated that two groups of conduction aphasics were discriminated: an efferent group with more expressive difficulty and anterior lesions, and an afferent group with less phonemic alterations but more comprehension problem, generally with more posterior lesions. However, clinical details of patients in the efferent group were not fully described. We consider that the characteristics of conduction aphasia were masked by nonfluent verbal output and defects in articulation in the acute stage in our case.

It remains unclear which lesion site is most important for the manifestation of conduction aphasia: the arcuate fasciculus, the insula, the superior temporal or supramarginal gyrus. There have been a few reported cases of conduction aphasia due to a lesion restricted to the arcuate fasciculus in the parietal operculum (Poncet et al., 1987; Tanabe et al., 1987). Since the damage to the insula was less extensive and the supramarginal gyrus was intact, the subcortical lesion interrupting the arcuate fasciculus may have been responsible for the occurrence of conduction aphasia. We could not, however, rule out the possibility that it might have been due to ischemic damage to the supramarginal gyrus on the basis of the ${ }^{123}$ I-IMP SPECT findings.

\section{REFERENCES}

Alajouanine T (1956) Verbal realization of aphasia. Brain, 79 $1-28$.

Benson DF (1979) Aphasia, Alexia, and Agraphia. Churchill Livingstone, New York.

Benson DF, Sheremata WA, Bouchard R, et al. (1973) Conduction aphasia: a clinicopathological study. Archives of Neurology, 28, 339-346.

Goodglass H and Kaplan E (1983) The Assessment of Aphasia and Related Disorders, 2nd Edn. Lea \& Febiger, Philadelphia.

Kertesz A (1984) Recovery from aphasia. In: Advances in Neurology, Vol. 42, Progress in Aphasiology (Ed. FC Rose), pp. 23-39. Raven Press, New York.

Kertesz A (1985) Aphasia. In: Handbook of Clinical Neurology, Vol. 45, Clinical Neuropsychology (Ed. JAM Fredericks), pp. 287-331. Elsevier, Amsterdam.

Mori E, Yamadori A and Hurumoto M (1989) Left precentral gyrus and Broca's aphasia: a clinicopathologic study. Neurology, 39, 51-54.

Poncet M, Habib M and Robillard A (1987) Deep left parietal syndrome: conduction aphasia and other neurobehavioural disorders due to a small subcortical lesion. Journal of Neurology, Neurosurgery, and Psychiatry, 50, 709-713.

Tanabe H, Sawada T, Inoue N, et al. (1987) Conduction aphasia and arcuate fasciculus. Acta Neurologica Scandinavica, 76, 422-427. 


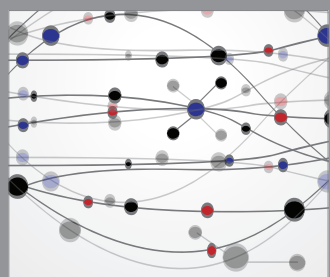

The Scientific World Journal
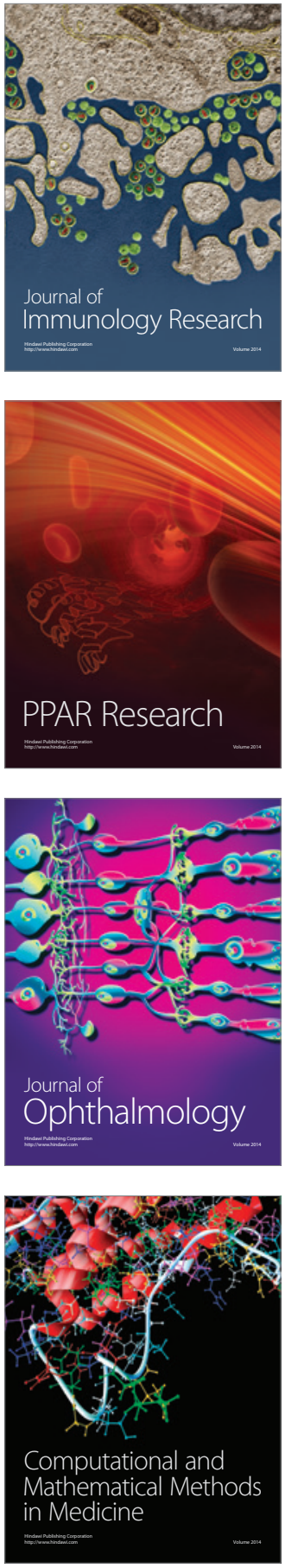

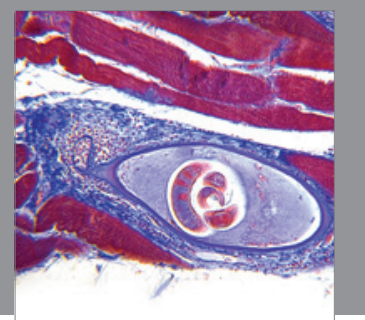

Gastroenterology

Research and Practice
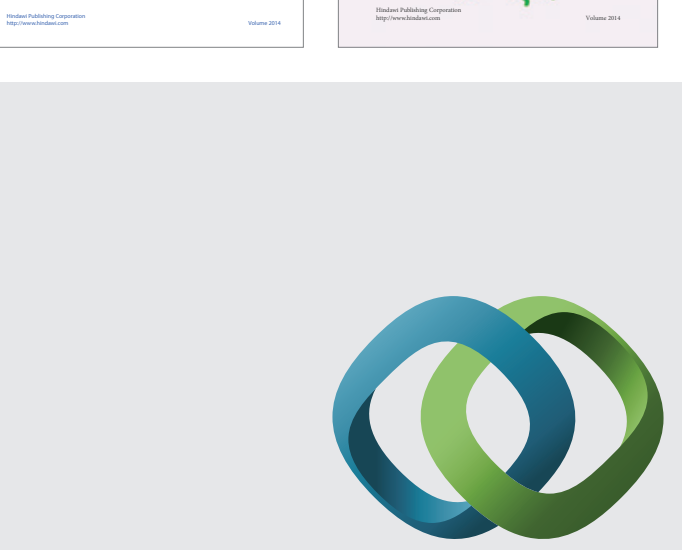

\section{Hindawi}

Submit your manuscripts at

http://www.hindawi.com
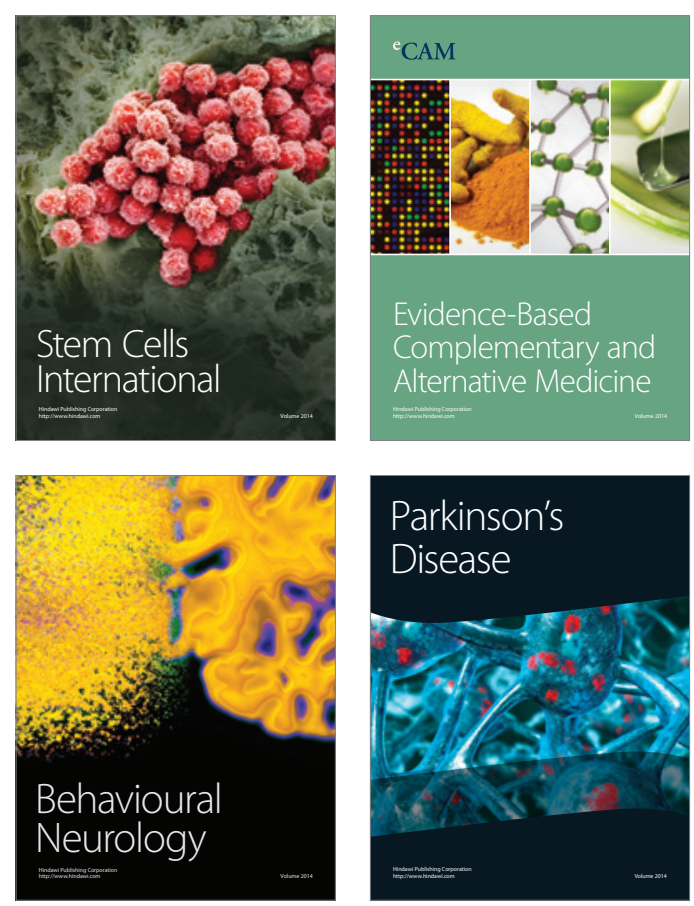

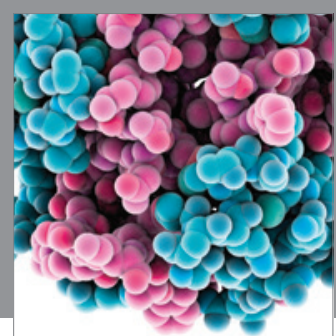

Journal of
Diabetes Research

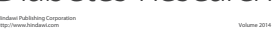

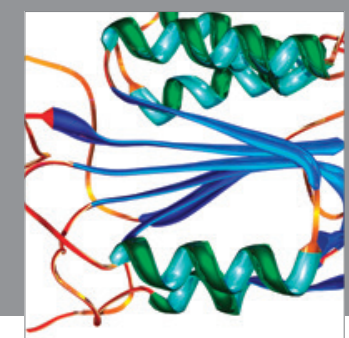

Disease Markers
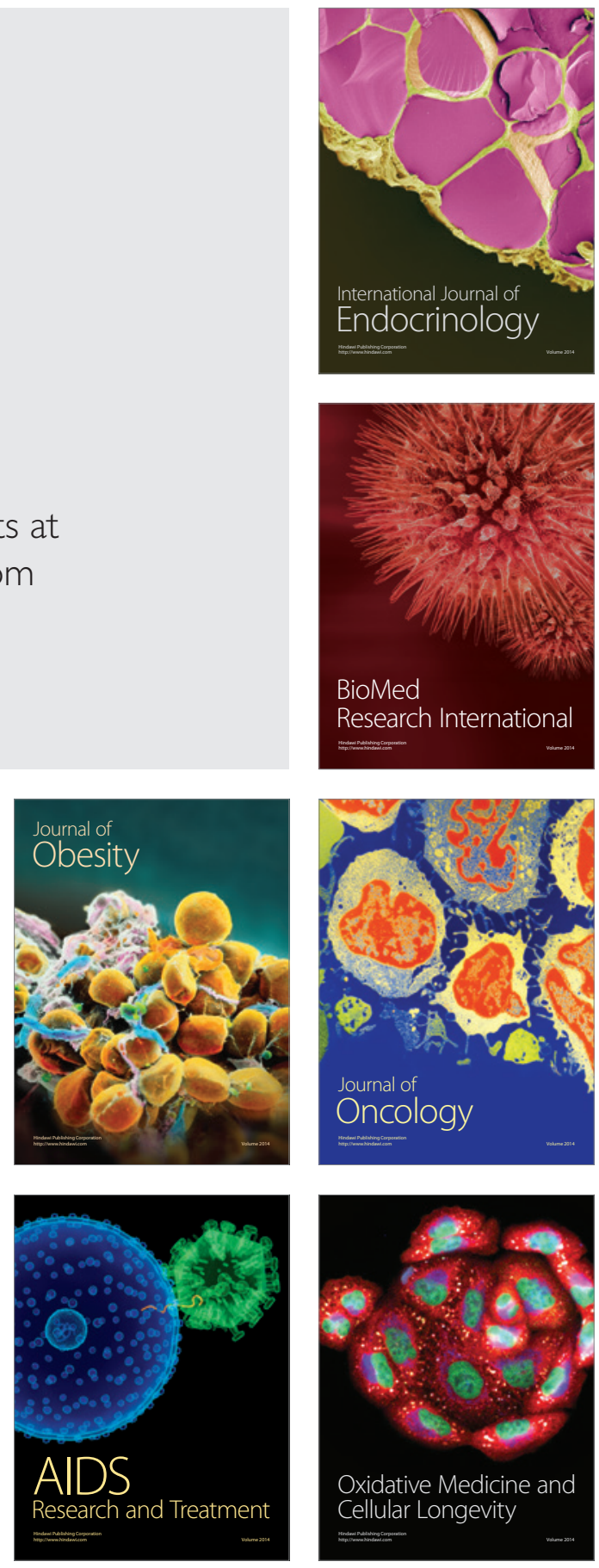